JAMA. 2019 September 17; 322(11): 1106-1107. doi:10.1001/jama.2019.10946.

\title{
Optimal Gestational Weight Gain
}

\author{
Lisa M. Bodnar, PhD, MPH, RD, \\ Department of Epidemiology, University of Pittsburgh Graduate School of Public Health, \\ Pittsburgh, Pennsylvania \\ Katherine P. Himes, MD, MS, \\ Department of Obstetrics, Gynecology, and Reproductive Sciences, University of Pittsburgh \\ School of Medicine, Pittsburgh, Pennsylvania
}

Jennifer A. Hutcheon, PhD

Department of Obstetrics and Gynaecology, University of British Columbia, Vancouver, Canada

\section{To the Editor}

The LifeCycle Project-Maternal Obesity and Childhood Outcomes Study Group compiled 196670 participant records from 25 cohorts to estimate optimal pregnancy weight gain ranges. ${ }^{1}$ We are concerned that the methods used to establish the optimal weight gain ranges have several serious shortcomings.

Optimal weight gain was based on a composite adverse outcome consisting of 7 equally weighted perinatal end points. This approach is problematic for several reasons. The composite components are associated with weight gain in different directions and vary in their importance to women and clinicians (eg, early preterm birth is more serious than cesarean delivery). Less severe outcomes occur the most; thus, the optimal weight gain range will be disproportionately driven by outcomes that are less important to women and clinicians.

In cardiovascular research, weighted outcomes have been used to produce more informative measures of disease burden ${ }^{2}$; a similar approach could have been used in this study. In addition, several key outcomes associated with weight gain were not included in the composite outcome, including perinatal death and postpartum weight retention. The strong positive effect of greater weight gain on weight retention alone could alter the reported optimal ranges.

The authors used an arbitrary $P$ value threshold for defining optimal gestational weight gain ("all weight gain categories with a statistically significant $[P<.05]$ protective association[OR $<1]$ for any adverse outcome"), but this is a problematic approach for

Corresponding Author: Lisa M. Bodnar, PhD, MPH, RD, Department of Epidemiology, University of Pittsburgh, 130 DeSoto St, 5129 Public Health, Pittsburgh, PA 15261 (bodnar@edc.pitt.edu).

Conflict of Interest Disclosures: Drs Bodnar and Hutcheon reported receiving grants from the National Institutes of Health. Dr Himes reported receiving grants from the National Institute of Child Health and Human Development and the University of Pittsburgh Clinical and Translational Science Institute pilot grant. Dr Hutcheon reported receiving grants from the Canadian Institutes of Health Research and a Canadian Research Chair. 
making policy or clinical decisions. ${ }^{3}$ A weight gain category with an odds ratio (OR) of 0.99 could be statistically significant, given a large enough sample size, even though a $1 \%$ risk reduction is unlikely to be clinically important.

Consequently, the optimal weight gain ranges could be excessively wide, failing to identify women who would benefit from a weight gain intervention. This may partially explain why the 2 broadest weight gain recommendations in the study occurred in the categories of body mass index with the most women (normal weight and overweight). Furthermore, using odds ratios or other relative measures of effect can cause physicians and patients to overestimate an intervention's benefit. ${ }^{4}$ The definition of optimal weight gain ranges should take the clinical significance of the findings into account, with the magnitudes of risk presented as absolute effect measures (such as risk differences).

Developing a rigorous, evidence-based public health recommendation for pregnancy weight gain requires an approach that considers the clinical importance of observed differences in risk, accounts for the relative severity and direction of the composite outcome constituents, and includes all outcomes of public health and clinical relevance.

\section{References}

1. Voerman E, Santos S, Inskip H, et al.; LifeCycle Project-Maternal Obesity and Childhood Outcomes Study Group. Association of gestational weight gain with adverse maternal and infant outcomes. JAMA. 2019;321(17):1702-1715. doi:10.1001/jama.2019.3820 [PubMed: 31063572]

2. Sampson UK, Metcalfe C, Pfeffer MA, Solomon SD, Zou KH. Composite outcomes: weighting component events according to severity assisted interpretation but reduced statistical power. J Clin Epidemiol. 2010;63(10): 1156-1158. doi:10.1016/j.jclinepi.2010.01.019 [PubMed: 20558037]

3. Amrhein V, Greenland S, McShane B. Scientists rise up against statistical significance. Nature. 2019;567(7748):305-307. doi:10.1038/d41586-019-00857-9 [PubMed: 30894741]

4. Akl EA, Oxman AD, Herrin J, et al. Using alternative statistical formats for presenting risks and risk reductions. Cochrane Database Syst Rev. 2011;3(3): CD006776. 